\title{
Effect of Levetiracetam Monotherapy on Serum Cortisol Levels - A Descriptive Study
}

\author{
M. Jeyakumar ${ }^{1}$, A. Kavitha², Jeyanthi Arulneyam³ \\ ${ }^{1}$ Department of Biochemistry, Velammal Medical College and Hospital and Research Institute, Madurai, Tamilnadu, \\ India. ${ }^{2}$ Department of Biochemistry, Velammal Medical College and Hospital and Research Institute, Madurai, \\ Tamilnadu, India. ${ }^{3}$ Department of Neuromedicine, Pondicherry Institute of Medical Sciences, Pondicherry, India.
}

\section{ABSTRACT}

\section{BACKGROUND}

Epileptic patients undergoing treatment with novel anti-epileptic drugs like Valproic acid and Phenytoin have increased serum cortisol levels. Levetiracetam is one of the second generations anti-epileptic drugs \& its effect on serum cortisol level was not well documented. So, we decided to measure serum Cortisol levels in newly diagnosed epileptic patients on Levetiracetam monotherapy before and six months after treatment.

\section{METHODS}

We included 60 newly diagnosed epileptic patients of primary cause starting on Levetiracetam monotherapy attending our Neurology OPD. Serum Cortisol levels were measured on the day of starting levetiracetam \& 6 months after starting the therapy and compared.

\section{RESULTS}

Study was carried out on 60 epileptic individuals, started on Levetiracetam monotherapy including 31(51.7\%) males and 29(48.3\%) females in the age group $27 \pm 7$ years. Baseline mean Cortisol level was $11.98 \pm 1.83 \mu \mathrm{g} / \mathrm{dl}$ and after 6 months it was $11.97 \pm 1.63 \mu \mathrm{g} / \mathrm{dl}$. There is no significant difference in cortisol level before and after 6 months of levetiracetam monotherapy.

\section{CONCLUSIONS}

Serum Cortisol levels remain unaltered after 6 months of Levetiracetam monotherapy which implies that Levetiracetam may not possibly affect serum cortisol levels. Further studies should be carried out to evaluate the long-term effect of Levetiracetam on serum Cortisol levels.

\section{KEY WORDS}

Serum Cortisol, Levetiracetam, Epilepsy, Anti-Epileptic Drugs
Corresponding Author:

A. Kavitha,

Tutor,

Department of Biochemistry,

Velammal Medical College and Hospital

\& Research Institute, Madurai- Tuticorin Ring Road, Anuppanadi, Madurai-625009,

Tamilnadu, India.

E-mail: kaviayanam@gmail.com

DOI: $10.14260 /$ jemds/2019/789

Financial or Other Competing Interests: None.

How to Cite This Article:

Jeyakumar M, Kavitha A, Arulneyam J. Effect of levetiracetam monotherapy on serum cortisol levels- a descriptive study. J. Evolution Med. Dent. Sci. 2019;8(49): 3649-3652, DOI: 10.14260/jemds/2019/789

Submission 03-10-2019, Peer Review 16-11-2019, Acceptance 23-11-2019, Published 09-12-2019. 


\section{BACKGROUND}

Epilepsy is one of the common and chronic neurological disorders characterized by unprovoked recurrent paroxysmal events or seizures associated with a sudden alteration in motor activity and behaviour with or without alteration in conscious awareness. ${ }^{1}$ Approximately $5-10 \%$ of the population will have at least one seizure, with the highest incidence occurring in early childhood and late adulthood. ${ }^{2} \mathrm{As}$ seizures and epilepsy are heterogeneous; they have to be classified for selecting appropriate drug therapy. International League against Epilepsy in 1981, divided seizures into partial and generalized. ${ }^{3}$ The type of medication used for epilepsy depends up on the empiric nature of the seizure. Hence considerable effort has been expended to classify seizures so that seizure diagnosis can be made, and, on that basis, appropriate therapy can be started. ${ }^{4}$ The decision to begin anticonvulsant therapy after a first seizure will be individualized for each patient. The decision varies based on the risk of additional seizures, the risk of seizurerelated injury, the loss of employment or driving privileges, and other psychosocial factors.

Monotherapy will be the treatment of choice. The classic antiepileptic drugs include phenobarbital, phenytoin, carbamazepine, valproic acid, benzodiazepines and ethosuximide. The newer anticonvulsant drugs include gabapentin, tiagabine, lamotrigine, topiramate, felbamate, zonisamide, oxcarbazepine and levetiracetam. These agents generally have less potential for drug interactions and fewer side effects than the older drugs. ${ }^{5}$

Levetiracetam (LEV) is a second-generation antiepileptic drug that can be used as a unique or adjunctive therapy for treating partial or generalized seizure. ${ }^{6}$ There has been increasing evidence that it can be used for patients with generalized absence or myoclonic seizure. ${ }^{7}$ Recently IV LEV is listed as a 'treatment option for the stage of established Status Epilepticus.'8

Cortisol is the major circulating glucocorticoid in humans, synthesized from cholesterol under the influence of ACTH in the zona fasciculata and zona reticularis of the adrenal cortex Steroidogenesis involves the concerted action of several enzymes; most of them belong to cytochrome P450 super familys. ${ }^{9}$ Both seizures and antiepileptic drugs (AEDs) may influence the brain areas involved in the regulation of the hypothalamus-pituitary function and affect its hormone secretion. Also, seizures may act as a "stressor" and activate the hypothalamus-pituitary-adrenal axis (HPAA). ${ }^{10}$

There is evidence pointing that treatment with antiepileptic drugs may increase serum Cortisol level.10,11 Levetiracetam is a new generation drug whose side effects and benefits are not fully explored. We would like to explore the effect of Levetiracetam monotherapy on serum Cortisol levels.

We wanted to estimate and compare the serum cortisol levels in epileptic patients receiving levetiracetam monotherapy at the time of initiation of therapy and 6 months after the therapy.

\section{METHODS}

This is a descriptive study conducted in the department of Biochemistry, Pondicherry institute of Medical sciences; Pondicherry during the period of January 2012 to August 2013. Ethical committee approval was obtained. We included 60 newly diagnosed epileptic patients of primary cause starting on Levetiracetam monotherapy in the age group 18 to 45 years attending PIMS Neurology OPD. Sample size was taken based on the convenience of the study. We excluded the patients with age group more than 45 years, patients with known history of Liver failure, Kidney disorders, known case of Cushing's disease, Addison's disease and patients currently on Steroids due to other medical reasons. The study protocol was explained to all the patients in their native language and written and informed consent was obtained. Blood samples were collected from the epileptic patients on the day of starting of Levetiracetam therapy and 6 months later. The dosage was unaltered for all these patients within 6 months. The patients whose dose was altered \& alternate AED was changed has been excluded from this study. After comforting the patients all the samples were collected at around 12 noon in all patients to avoid circadian rhythm effect of Cortisol. Serum was separated and Cortisol levels were measured by Electrochemiluminescence Immunoassay method using Cobas Integra e411 autoanalyser.

\section{Statistical Analysis}

Continuous variables were expressed as mean \pm standard deviation. Paired $t$ test, a parametric test was used to compare the mean value of the parameters in epileptic patients on the day of starting of therapy and 6 months after the therapy. $p$ value of $\leq 0.05$ was accepted as statistically significant. SPSS version 17.0 software was used.

\section{RESULTS}

The study was carried out on 60 epileptic individuals started on Levetiracetam monotherapy. It included 31(51.7 \%) males and $29(48.3 \%$ ) females (figure 1$)$ in the age group $27 \pm 7$ years. The baseline characteristics were shown in table 1 .

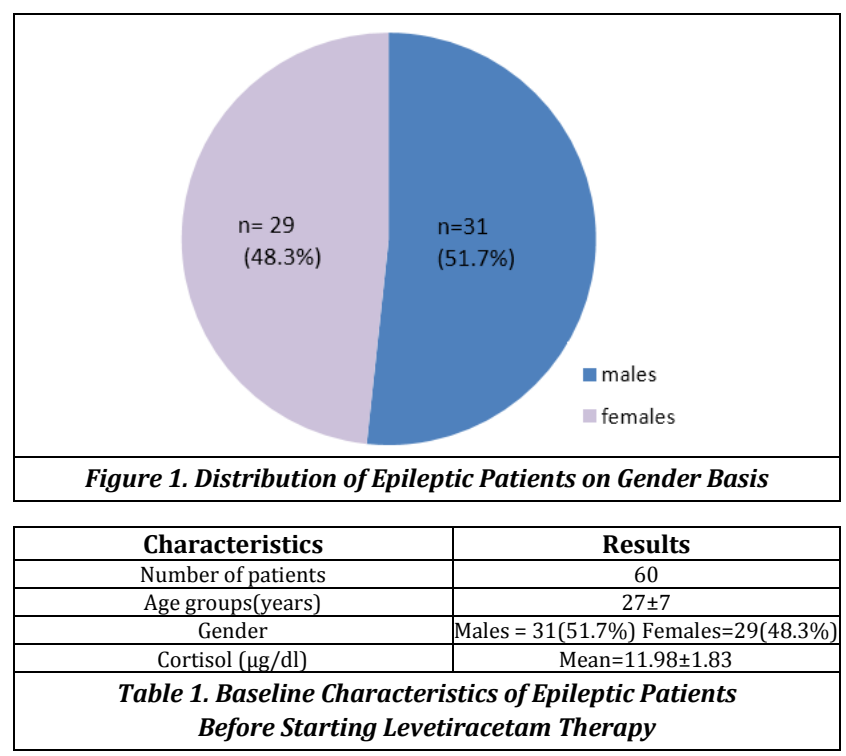




\begin{tabular}{|c|c|c|c|}
\hline Parameter & Baseline & After 6 months & p-Value \\
\hline Serum Cortisol $(\mu \mathrm{g} / \mathrm{dl})$ & Mean $=11.98 \pm 1.83$ & Mean $11.97 \pm 1.63$ & 0.9 \\
\hline \multicolumn{3}{|c|}{ Table 2. Comparison of Cortisol in Epileptic Patients before } \\
and after 6 Months of Treatment Using Paired t Test \\
\hline p-value $<0.05$ - statistically significant
\end{tabular}

\begin{tabular}{|c|c|c|}
\hline & & Differences in Cortisol Levels $(\mu \mathrm{g} / \mathrm{dl})$ \\
\hline \multicolumn{2}{|c|}{ Mean } & 0.005 \\
\hline \multicolumn{2}{|c|}{ S.D. } & 0.72 \\
\hline \multicolumn{2}{|c|}{ Minimum } & -1.90 \\
\hline \multicolumn{2}{|c|}{ Maximum } & 3.00 \\
\hline \multirow{2}{*}{ Percentiles } & $25^{\text {th }}$ & -0.40 \\
\hline & $75^{\text {th }}$ & 0.47 \\
\hline \multicolumn{3}{|c|}{$\begin{array}{c}\text { Table 3. Difference in Serum Cortisol of Epileptic Patients } \\
\text { before and after Treatment }\end{array}$} \\
\hline
\end{tabular}

There is no statistically significant difference in serum cortisol levels before and 6 months after Levetiracetam monotherapy (tables 2 \& 3 )

\section{DISCUSSION}

In recent years there has been increasing evidence suggesting that epilepsy and its treatment can affect serum Cortisol levels. Novel anti-epileptic drugs like Valproic acid and Phenytoin may increase Cortisol levels. Aydin et al in 2005 found that serum cortisol is elevated in epileptic patients receiving AED and they related this elevated cortisol with weight gain. ${ }^{11}$ Galimberti CA et al have reported that serum cortisol levels were increased in epileptic women receiving anti-epileptic drugs and the cortisol level were correlated with seizure frequency. ${ }^{12}$ The mechanism underlying these changes was found to be the close relationship with the alteration of signals in the hypothalamic-pituitary axis. The pituitary gland is responsible for releasing adrenal cortical hormones, sex hormones, including FSH and LH, whereas the hypothalamus indirectly controls these hormones by releasing ACTH \& gonadotropin-releasing hormone that targets receptors in the pituitary gland. During epileptic discharge, altered signals from the cerebral cortex, amygdala and hippocampus reaches the hypothalamus through GABA and glutamate as mediators. ${ }^{13}$ Some AEDs associated with the Cytochrome p450 enzyme system in the liver found to affect these hormone levels.

Only little is known about the effect of Levetiracetam on serum Cortisol levels. So, we measured the levels of serum cortisol before and after 6 months of levetiracetam monotherapy. In 2008, Gustavsen et al investigated the possible effects of valproate, carbamazepine and levetiracetam on steroidogenesis using the H295R steroidogenesis model. They observed that there is no significant dose-response effect on hormone production with carbamazepine or Levetiracetam. ${ }^{14}$ To best of our knowledge, this is the first human study to evaluate the effect of LEV on serum cortisol levels. By this study we found that there is no statistically significant difference in cortisol level before and after 6 months of levetiracetam monotherapy. This may be due to the fact that LEV has got unique pharmacokinetic property \& its mechanism of action is not by targeting any of the known neurotransmitters. The main metabolic pathway of LEV was found to be through hydrolase acetyl animation transformation. The metabolite (UCB 1057) produced will not be transformed by liver cytochromes and thus LEV and its main metabolites do not affect the activity of hepatic cytochromes $\mathrm{p} 450$ enzyme system, ${ }^{15}$ whereas the novel AEDs affects the liver p450 enzyme system. Another possible explanation is that LEV shows its anti-epileptic effect by binding with an integral membrane protein present on all synaptic vesicles called SV2. SV2 is a small gene family consisting of three isoforms, designated SV2A, SV2B, and SV2C.

The function of SV2s is found to regulate presynaptic calcium levels during repetitive activity or SV2s functions as targets for residual calcium in regulating vesicular exocytosis. SV2A is the most widely distributed isoform, being nearly ubiquitous in the CNS, as well as being present in endocrine cells. ${ }^{16}$ SV2B is brain specific, with a wide but not ubiquitous distribution, and SV2C is a minor isoform in brain. By immunocytochemistry, it is found that SV2A is the major binding sites of Levetiracetam. It is found that SV2A may regulate priming of docked synaptic vesicles, a process that makes them ready for exocytosis, and thus selectively enhancing low frequency neurotransmission, ${ }^{7}$ whereas other novel AEDs target ion channels or inhibitory neurotransmitter receptors. ${ }^{17,18}$

Our study has some limitations. First of all, the study group we included is relatively small. Also, we assessed only the early effects of levetiracetam on the serum cortisol of epileptic patients. To assess the later effects of this drug we need to have longer follow up period.

\section{CONCLUSIONS}

Levetiracetam is unlikely to alter the serum cortisol levels even after 6 months of treatment. Further prospective studies involving large group of population and longer follow-up period should be carried out to explore the effect of this drug on serum cortisol and other related hormones.

\section{REFERENCES}

[1] Fisher RS, van Emde BW, Blume W, et al. Epileptic seizures and epilepsy: definitions proposed by the International League Against Epilepsy (ILAE) and the International Bureau for Epilepsy (IBE). Epilepsia 2005;46(4):470-2.

[2] Fauci AS, Braunwald E, Kasper DL, et al. Harrison's principle of Internal medicine. Chapter - 363. Seizure \& epilepsy. 17th edn. New Delhi: McGraw-Hill Publisher 2008: p. 2498.

[3] Abou-Khalil B. Levetiracetam in the treatment of epilepsy. Neuropsychiatric Disease and Treatment 2008:4(3):507-23.

[4] Katzung BG, Master SB, Trevor AJ. Basic and clinical pharmacology. Chapter - 24. Antiseizure drugs. 11 th edn. New Delhi: McGraw-Hill Publication 2009: p. 399.

[5] Ghosh AK. Mayo clinic internal medicine review. Chapter - 19. Neurology. $8^{\text {th }}$ edn. Canada: CRC Press 2008: p. 755. 
[6] Stepanova D, Beran RG. Measurement of levetiracetam drug levels to assist with seizure control and monitoring of drug interactions with other Anti-Epileptic Medications (AEMs). Seizure 2014;23(5):371-6.

[7] Gambardella A, Labate A, Colosimo E, et al. Monotherapy for partial epilepsy: focus on levetiracetam. Neuropsychiatric Disease and Treatment 2008:4(1):338.

[8] Trinka E, Dobesberger J. New treatment options in status epilepticus: a critical review on intravenous levetiracetam. Therapeutic Advances in Neurological Disorders 2009;2(2):79-91.

[9] Ganong FW. The adrenal medulla \& adrenal cortex review of medical Physiology. 21 ${ }^{\text {st }}$ edn. Lange 2003:51352.

[10] Pritchard PB 3rd. The effects of seizures on hormones. Epilepsia 1991;32(Suppl 6):S46-50.

[11] Aydin K, Serdaroglu A, Okuyaz C, et al. Serum insulin, leptin and neuropeptide y levels in epileptic children treated with valproate. J Child Neurol 2005;20(10):84851.

[12] Galimberti CA, Magri F, Copello F, et al. Seizure frequency and cortisol and dehydroepiandrosterone sulfate (DHEAS) levels in women with epilepsy receiving antiepileptic drug treatment. Epilepsia 2005;46(4):51723.
[13] Montouris G, Morris GL 3rd. Reproductive and sexual dysfunction in men epilepsy. Epilepsy Behav 2005;7(Suppl 2):S7-14.

[14] Gustavsen, Wendel M. Reproductive endocrine side effects of antiepileptic drugs, 2008. http://urn.nb.no/URN:NBN:no-19139.

[15] Xiaotian X, Hengzhong Z, Yao X, et al. Effects of antiepileptic drugs on reproductive endocrine function, sexual function and sperm parameters in Chinese Han men with epilepsy. J Clin Neurosci 2013;20(11):1492-7.

[16] Buckley K, Kelly RB. Identification of a transmembrane glycoprotein specific for secretory vesicles of neural and endocrine cells. J Cell Biol 1985;100(4):1284-94.

[17] Crowder KM, Gunther JM, Jones TA, et al. Abnormal neurotransmission in mice lacking synaptic vesicle protein 2A (SV2A). Proc Natl Acad Sci USA 1999;96(26):15268-73.

[18] Custer KL, Austin NS, Sullivan JM, et al. Synaptic vesicle protein 2 enhances release probability at quiescent synapses. J Neurosci 2006;26(4):1303-13. 